\title{
A Kind of Smart Home Electricity Controller based on Active Distribution Networks and ATT7035 Chip
}

\author{
Lefeng Cheng ${ }^{a}$, Maoxin $\mathrm{Xu}^{\mathrm{b}}$, Tao $\mathrm{Yu}^{\mathrm{c}}$, Yixuan Chen ${ }^{\mathrm{d}}$, Baomin Zheng ${ }^{\mathrm{e}}$ \\ College of Electric Power, South China University of Technology, Guangzhou 510640, China \\ a lefengg@126.com, ${ }^{\text {b } 970579382 @ q q . c o m, ~}{ }^{c}$ taoyu1@scut.edu.cn, ${ }^{\text {d } 376100073 @ q q . c o m, ~}{ }^{\text {e }}$ \\ 2420535338@qq.com
}

Keywords: Active distribution network (ADN); smart grid; smart controller; ATT7035; electricity information collection; ZigBee communication technology

Abstract. The distribution network may be influenced by access of a large number of distributed generations, such as the impact of short level, reactive power, voltage ride through limitation, bi-directional power flow, etc., which brought a lot of uncertainty for networks' operation and controlling. The future development mode and key direction of distribution network is transformed from passive control to active control. Based on this, a smart home electricity-using controller is designed and developed according to the user layer of active power distribution, which takes ATT7035 chip as core controller, can measure voltage, current, active power, active power and power factor of home appliance, and also calculate the cumulative active energy consumption; the calculation results can be transferred to the smart interactive terminal of home system through ZigBee technology, then the home appliance can be opened and closed by the designed controller through real-time instructions of the smart interactive terminal. The core CPU system controlling module, power drive module, and power parameters acquisition and conditioning module, ZigBee communication module of the smart controller is designed in this paper, and some of their hardware principle diagrams are given. The designed controller has advantages of multiple functions, high reliability, and strong practicability etc., and has a certain market application prospect.

\section{Introduction}

With the continuous improvement of penetration of distributed energy resource (DER) on all levels of power system, the power system has become relatively complex, especially its planning and operation mode, while also a great influence has been made on economic and regulatory approaches of distribution network ${ }^{[1]}$. In response to a series of changes such as gradually expansion of DER scale and the user's increasing expectations of power supply reliability within a reasonable price range, traditional distribution network has gradually transformed from a passive mode to active mode ${ }^{[2]}$. Active distribution network which is mainly aimed at resolving the problems of power grid distribution network compatibility and the application of large-scale intermittent renewable energy, and whose goal is to increase the green energy utilization efficiency and optimize primary energy structures and so on ${ }^{[3]}$. Aimed at the influence of DER access into distribution network such as effecting the short-circuit level, reactive power and voltage distribution, etc. , especially when the new energy sources are accessed in a plugging and playing mode, which brings more uncertainties to the operation of network and making it more difficult to control ${ }^{[4]}$.

Electricity utilization controlling method is diverse, including both simple breaking control and the most advanced real-time feedback controlling mode. Currently, the vast majority of home intelligent controllers in the market have only minimal intelligence, such as with the function of turning in and off the light at regular time, but it cannot realize monitoring and power off controlling aimed at abnormal situation, thus doesn't have real intelligent features and the price is very expensive. Existing home appliances sockets have been unable to meet various needs, especially the active smart home system based on the distribution network and which needs intelligent controllers with innovative features and large potential market demand ${ }^{[5,6]}$. Hence, a smart home power controller is developed based on the core information processing chip ATT7035 chip in this paper. 
This controller can measure the voltage, current, frequency, active and reactive power, power factors of electrical equipment (refrigerators, washing machines, microwave ovens, etc., shown in Fig.1), and it can calculate the active power accumulated consumption, after obtaining the measurement and calculation results, it provides electric energy data to intelligent interactive terminals in the smart home systems (Andrews smart phone, tablet, etc.) through ZigBee communication module and the upper software APP on the intelligent interactive terminals can issue commands based on the real-time electric energy data to the intelligent controllers, once the intelligent controllers receive responding commands from interactive terminals, the relay modules in smart electricity sockets are controlled to realize turning on and closing off, thus can control the home appliances which are connected to the smart electricity sockets.

In addition, this home intelligent controller can also achieve self-protection by means of the protection modules, such as lightning protection, overvoltage protection, overcurrent protection, short circuit protection, time opening, time closing, automatic alarm, automatic sleep, status indication and other intelligent functions.

\section{Hardware design}

The smart electrical controller is designed based on the core chip ATT7035 CPU controlling system module, as well as concluding power drive controlling module, interface module, clock module, reset circuit, LCD display module, ZigBee communicaiton module, power parameters acquisition and conditioning module, switch circuits, etc., which is shown in Fig.1. ATT7035 chip is the core controlling unit, which can process each detection signal and keyboard input value, and the results can be displayed on the LCD, and through the action of relays in switch circuit the home applicance equipment connected to the smart sockets can be turned on and closed off. The power drive controlling module adopts the scheme of transformer step-down voltage + DC regulators, which can provide power to the LCD display module and CPU module. The ATT7035 core controlling chip is a dedicated single-phase SOC (system-on-chip) energy measurement chip, which is integrated with processor unit, single-phase electric energy metering unit, power management, clock management and PLL multiplier and JTAG debug features. The ATT7035 chip's function module diagram is shown in Fig.2.

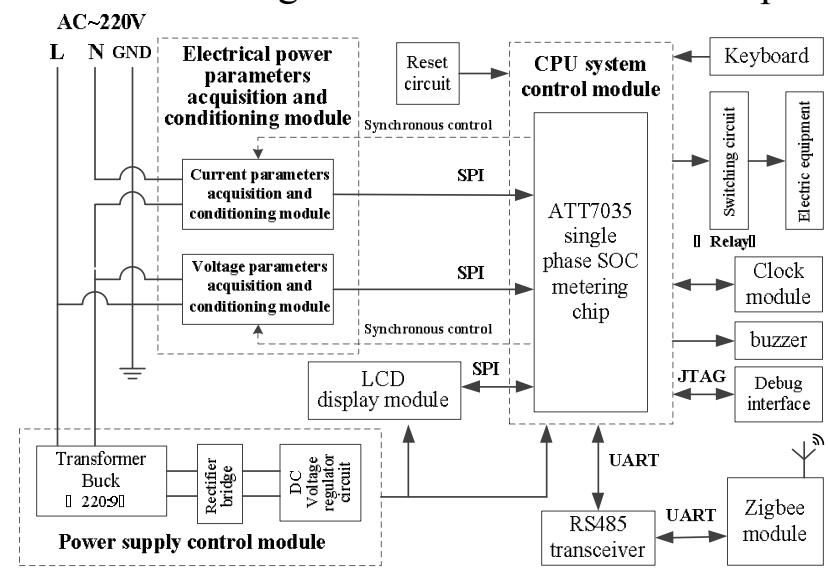

Fig.1 The smart controller hardware principle diagram

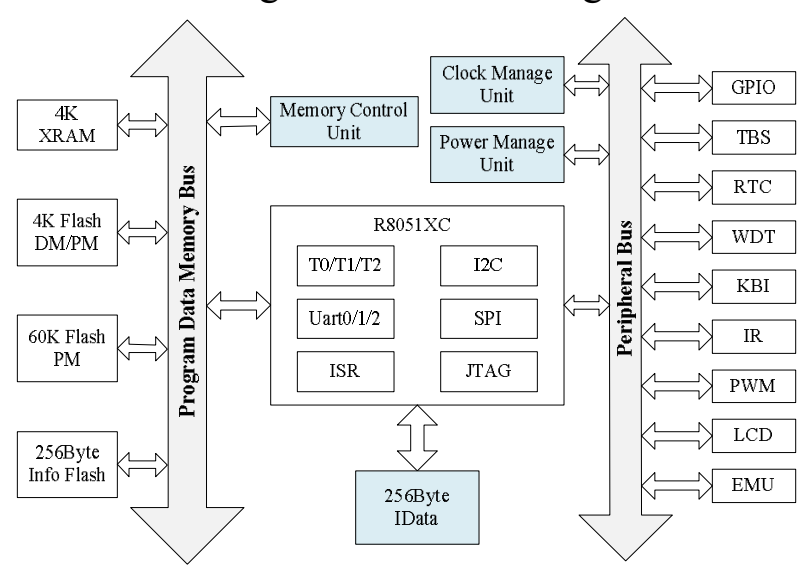

Fig.2 The ATT7035 chip's function module diagram

Basic parameter indexes of designed smart home electricity controller. In this paper, the basic parameter indexes are: $220 \mathrm{~V}$ AC input voltage, can work normally under $45 \sim 60 \mathrm{~Hz}$; the largest load current Imax is $15 \mathrm{~A}$; power loss is smaller than $1 \mathrm{~W}$; precision aspects: voltage (1.0 grade) smaller $1 \%$, current (1.0 grade) smaller $1 \%$, power ( 1.0 grade) smaller $1 \%$, electric energy ( 1.0 grade) smaller $1 \%$; low temperature operating conditions: $-10^{\circ} \mathrm{C} \pm 2^{\circ} \mathrm{C}$, can work properly for $2 h$; high tempertare operating conditions: $+50^{\circ} \mathrm{C} \pm 2^{\circ} \mathrm{C}$, can work properly for $2 h$.

Power drive controlling module. This module can implement AC-DC conversion on the $220 \mathrm{~V}$ AC voltage, in this paper, the DC working voltage used in the designed smart controller is $5 \mathrm{~V}$ and $3.3 \mathrm{~V}$, 
the $5 \mathrm{~V}$ can provide the voltage to the parameter acquisition and conditioning module, clock module and display module which are shown in Fig.1 and the latter can provide voltage to the CPU core controlling module, ZigBee wireless communication module, and also the electromagnetic compatibility performance is took into consideration. At present, there are mainly three kinds of technical scheme to realize AC-DC transformation in industry, such as switch power module scheme, resistance capacitance step-down scheme and transformer step-down rectification scheme. Among them, the switch power module has advantages of small volume, high efficiency and strong anti-interference ability, but the output power is not isolated and safe, cannot be used for large power $\operatorname{load}^{[7]}$. So this paper chooses the transformer (the ratio of 220:9) to lower voltage and then rectify and filter, the rectifier is composed of four diodes. Seen in Fig. 3 bellow, which is the design schematics of power drive module, the $220 \mathrm{~V}$ AC voltage is lowered to $9 \mathrm{~V} \mathrm{AC}$ voltage, and then after the action of rectifying circuit (D1 D4), capacitor filtering (C2), the signal is transmitted to power chip LM7805 to output $+5 \mathrm{~V}$ DC voltage and then after filtering of capacitor $(\mathrm{C} 3 / \mathrm{C} 4)$, the signal is transmitted to power chip LM1117L3 and once through the filtering of capacitor $(\mathrm{C} 5 / \mathrm{C} 1)$, finally $\mathrm{DC}+3.3 \mathrm{~V}$ is output.

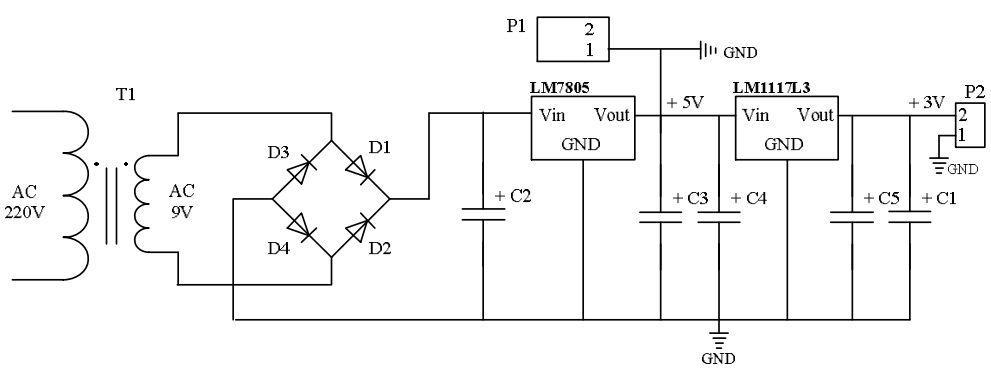

Fig.3 The power driver module circuit diagram

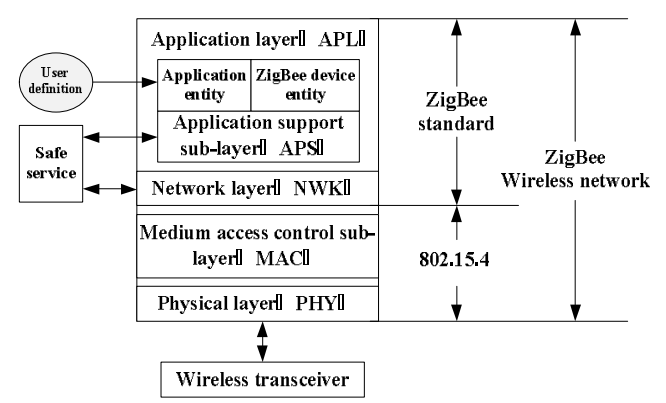

Fig.4 The model of ZigBee protocol

Electrical energy parameters acquisition and conditioning module. This part is designed to implement acquisition and conditioning on power parameter measurement signal, which plays a very important role in the whole design of home electricity intelligent controller. Core control chip ATT7035 has special requirements that the input voltage of V3P and V3N pin of voltage signal acquisition terminal must between $-700 \mathrm{mV}+700 \mathrm{mV}$, so the voltage signal collected should through the resistor voltage divider and then be introduced to the ATT7035 chip pins, then the high voltage signal can be converted into small voltage signals, and then input to the special measurement chip, in which has $\mathrm{A} / \mathrm{D}$ conversion and digital operation amplification circuit, thus can automatically complete the multi-channels voltage and current effective value and power calculation. The current signal is collected based on the principle of Ohm's law $I=U / R$, the voltage signal is converted into current signal, and the $I(\mathrm{t})$ is sent to V1P and V1N input port of ATT7035 through RC low pass filter.

ZigBee wireless communication module. The network topology structures which are supported by ZigBee wireless communication technology are: star, mesh, and cluster shape, each kind of network topology has its respective advantage and disadvantage ${ }^{[8,9]}$, and we should select them according to actual application ${ }^{[9]}$. The model of ZigBee protocol is shown in Fig.4 above.

The intelligent controller designed in this paper is set in application service level which can be applied in the smart home system, according to family living environment complexity and intelligent home system functional diversity, generally, the network mode of system can be adopted cluster shape network, which is usually consists of a ZigBee coordinator, several ZigBee network routers and some network terminals. In this paper, the designed communication scheme is realized by serial communication with ZigBee module through the UART interface and outside connection RS485 transceiver, the ZigBee module receives controlling commands from the interactive terminal and executes them and the response information is transmitted through wireless mode to the interactive terminal in the smart home system. 


\section{Power calculation and anti-electric energy stolen scheme design}

Power and effective value (RMS) conversion calculation. Active power, reactive power and apparent power output registers are available in the ATT7035 chip. The power and RMS value can be displayed directly by multiplying the corresponding coefficient $\mathrm{Kx}$, in order to test $\mathrm{Kx}$ is whether correct, we can use the following reference conversion formula. At the same time, the following figure 5 shows the power calculation and compensation of flow chart. The active power conversion formula is

$$
P_{x}=\frac{P_{r e g} * 51840 * 10 \wedge 6}{2 \wedge 23 * E C^{*} H F C o n s t}(\mathrm{~W})
$$

Where $P_{\text {reg }}$ is a power value read from the power register PowerP; $E C$ is a pulse constant of the intelligent controller; HFConst is the number of output pulses of the HFConst register in ATT7035. The reactive power convension calculation formula is

$$
Q_{x}=\frac{Q_{\text {reg }} * 51840 * 10^{\wedge} 6}{2 \wedge 23 * E C * H F C o n s t}(\text { Var })
$$

Where $Q_{\text {reg }}$ is a power value read from the power register PowerQ; $E C$ is a pulse constant of the intelligent controller; HFConst is the number of output pulses of the HFConst register in ATT7035.

IRMS conversion formula is

$$
I_{x}=\frac{I_{t}}{\operatorname{Ris}^{*} \operatorname{Gain}^{*} 1.11 * 2 \wedge 23}(\mathrm{~A})
$$

Where $I_{t}$ is the current effective value data read from IRMS; Ris is a current sampling resistance value; Gain is the gain of the current channel.

URMS conversion formula is

$$
U_{x}=\frac{U_{t}^{*} R_{t}}{\text { Rus }^{*} \operatorname{Gain}^{*} 1.11^{\wedge} 23}(\mathrm{~V})
$$

Where $U_{t}$ is the voltage effective value read from the URMS; $R_{t}$ is the total series resistance value of voltage channel; Rus is a voltage sampling resistance value; Gain is the gain of the voltage channel.

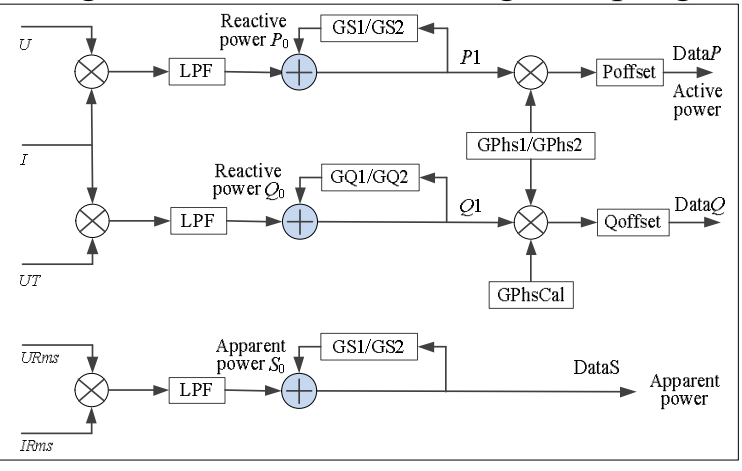

Fig.5 power calculation and compensation flow

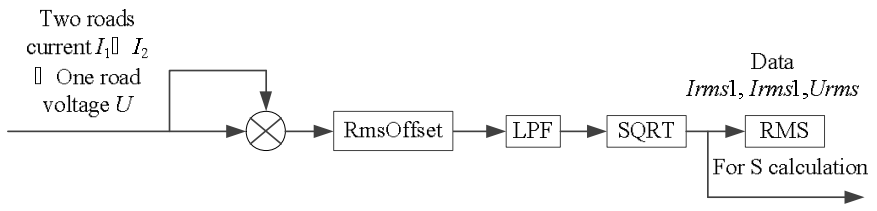

Fig.6 The voltage and current RMS calculation flow chart

As is shown in Fig.5 above, the active power $P$ value is got by multiplying the voltage $U$ and the current $I$ together after the low-pass filter. When calculating the reactive power $Q$, the voltage phase shifts $90^{\circ}$ at first, and then multiply the voltage $U$ and the current $I$ through the phase shifter, and finally the $Q$ value will be obtained through the low-pass filter LPF. The apparent power $S$ is obtained by multiplying the voltage effective value $U R m s$ and the current effective value IRms. The Channel 1 and Channel 2 provide a power gain correction and a phase correction respectively, and an offset correction is provided to eliminate outside interference for improving the accuracy of small signals. The Filter can make a voltage phase shift of $90^{\circ}$ and its performance is associated with the input signal frequency and the ADC sampling rate. The reactive phase compensation register $\mathrm{QPhsCal}(49 \mathrm{H}) \mathrm{can}$ make a phase compensation to the phase shift of $90^{\circ}$. The QPhsCal defaults to 0 , and when the femu is $505296 \mathrm{MHz}$, the corresponding input signal of $50 \mathrm{~Hz}$ can make an accurate phase shift of $90^{\circ}$.

Voltage and current RMS calculation and voltage frequency output. The ATT7035 chip can output two current signals $I_{r m s 1}$ and $I_{r m s 2}$ and one RMS voltage signal $U_{r m s}$, whose RMS accuracy is 
guaranteed $0.5 \%$. At the same time, it can output voltage frequency by way of zero-to-peer technology, and frequency accuracy can be guaranteed to $0.01 \mathrm{~Hz}$. The voltage and current RMS calculation flow chart is shown in Fig.6 above.

Anti-electric energy stolen scheme design. In ATT7035 chip, the anti-electric energy stolen module can compare the value of two channels of current signal, and then select the larger one channel for calculation. The FLTON (52H.5) is provided to set wether the anti-electric energy stolen module is turned on. If FLTON $=0$, the user can select the channel according to CHNSEL(51H.4), or if FLTON $=1$, the anti-electric energy stolen module automatically selects the appropriate measurement channel according to user's settings. Users can also select two currents to make a vector addition through CIADD $(51 \mathrm{H} .3)$, and then calculate it. The user can set the electricity-stolen ratio by the ICHK $(50 \mathrm{H})$. Such as, you can set $0 \times 10 \mathrm{H}$ to represent when the difference of two current RMS reach $6.25 \%$, it shows that the electricity is stolen. When the RMS of two channels current signal are less than the value of ITAMP $(59 \mathrm{H})$, Channel 1 will be selected as the default measurement unit to prevent the interference of small signal noise. When I2GT1 (51H.5) shows 0 , it means I1 is higher than I2, and 1 means I2 is higher than I1. The Fig. 7 shows the automatic anti-electricity stolen scheme design.

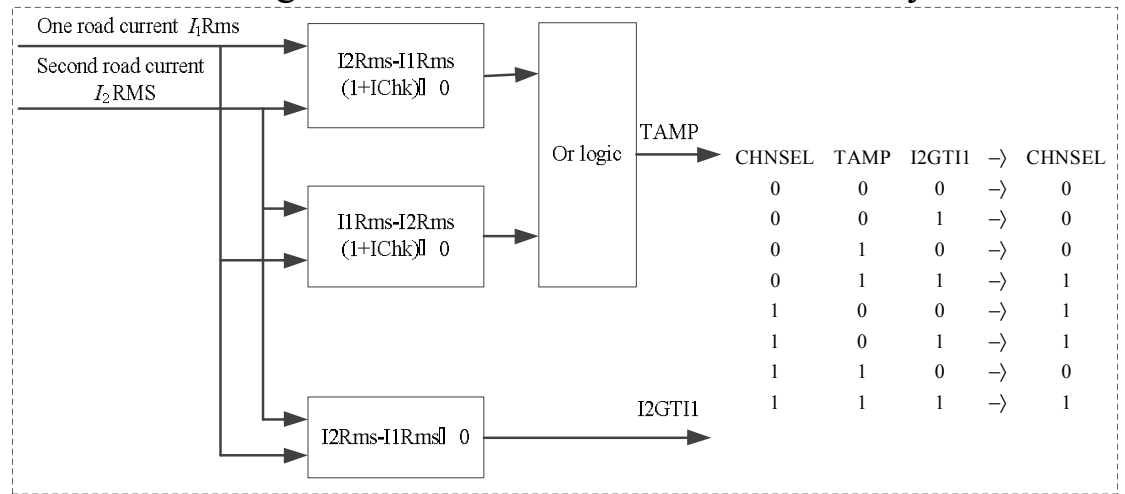

Fig.7 Automatical preventing electricity-stolen scheme

When TAMP (51H.6) shows 1, it indicates the electricity is stolen and that is, two channels of current are over the setting anti-electricity stolen threshold value. The automatical anti-electricity stolen can be set as follows.

a) Open the ADC2 channel through the EMU_Ctr (5BH.5): Adc_I2on=1;

b) Correct the output value of channel 2 through I2GAIN (4AH) to make sure the output RMS of the two channels are consistent under the same input current, and then calculate the power of the second signal : PPXEN $=1$;

c) According to the anti-electricity stolen threshold vaule in need, set $\mathrm{ICHK}(50 \mathrm{H})$, and the setting is based on RMS (tampsel $=0$, default) or active (tampsel $=1$ );

d) Set the ITAMP $(59 \mathrm{H})$ according to the minimum detected current or power value of anti-tampering;

e) Sorrect the output value of channel 2 through I2GAIN to ensure the output RMS of the two channels are consistent under the same input current;

g) Open the anti-electricity stolen function, set FLTON (0x52H.5) 1 to open the function of automatic anti-electricity stolen.

After the automatic anti-electricity function is opened, the CHNSEL and CIADD are in a read-only state, and the channel selection is determined by the results of anti-electricitiy stolen, and we can check the tamper status through the register bits CHNSEL/TAMP/I2GTI1.

\section{Hardware system software design}

During the software design of ATT7035, the work mainly including software writing and simulation debugging. The single-phase energy metering IC ATT7035 chip is designed based on CPU52 with a 8052-compatible instruction set and a bus structure. The simulation platform of ATT7035 chip is MedWinV3, which currently can be only installed and run with Windows XP system ${ }^{[10]}$. Before 
installing MedWinV3 simulation platform, the Keil compiler environment should be installed to use C language for programming. The compilation is qualified only means there is no syntax errors in source program. As for other errors that exist in the source, you need to find out through repeated simulation and debugging ${ }^{[1]}$. The simulation means implement debugging, investigation and inspection on the target and it is generally divided into two kinds: hardware simulation and software simulation. Fig. 8 is a schematic view of hardware emulation. Hardware emulation means making an online simulation through simulator and the target prototype. The simulator provides signals and datas to the application circuit of the target prototype for testing and debugging. And it can run the program by single-step, continuous implementation or other ways, and it can also observe the changes in the ATT7035, which making it easy to modify the program errors. Fig. 9 is a main flow chart of the software system.

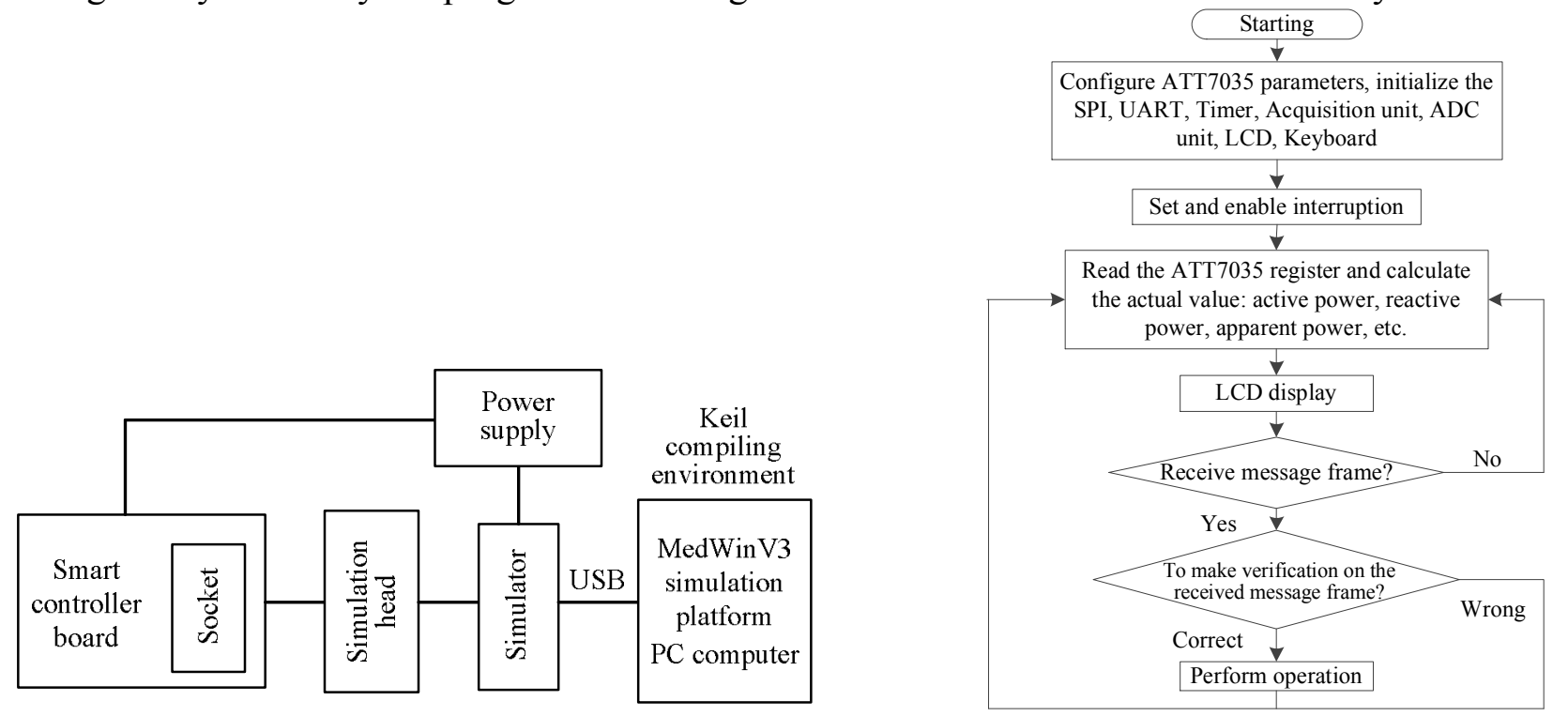

Fig.8 Hardware emulation mode diagram

Fig.9 The main program flow chart

This design of household appliances intelligent controller achieve many functions, including energy parameter acquisition and display, the wireless communication and transmission of electrical energy data, turning on and off the relay, over-current and over-voltage protection, leakage protection and automatic alarm. The debugging program of the software program mainly includes $\mathrm{I}^{2} \mathrm{C}$ program, and it is responsible for reading and writing the data stored in the chip $\mathrm{I}^{2} \mathrm{C}$ to ATT7035 chip. The LCD procedures program is mainly responsible for the LCD display. The clock program is mainly for reading and displaying calendar time and setting the calendar. The metering program is mainly used to calculate the cumulative energy and active power, pack and sent the energy data, read and process the energy data, etc. The timer program is primarily used for timing. The system program is mainly for writing the power ratio to the chip $\mathrm{I}^{2} \mathrm{C}$ and reading power ratio from the chip $\mathrm{I}^{2} \mathrm{C}$. The main program is mainly used for system initialization. Its main function calls other functions to achieve the full function of the intelligent controller. The main program also includes various types of header files, which mainly define register and its pins and related functions and declare variables. Of course, for this intelligent controller,some programs and functions of the software system can be design in many other ways, thus they can be further optimized.

\section{Conclusions}

This paper is written based on a large background that the current distribution network is existing in transition process from passive control to active control, aimed at the household electrical controller in smart home system, a smart home electricity utilization controller was developed based on ATT7035 core controlling chip.

(1) Which could measure the voltage, current, frequency, active and reactive power and power factor of domestic electric appliances, and as well as could calculate the power consumption of active power, after obtaining measuring and calculating results, which could be provided to the smart 
interactive terminals of intelligent home system through the ZigBee communication module, while the smart controller waited the controlling commands from interactive terminals in real-time, further, the opening and closing condition of smart controllers could be controlled, thus controlled the home electric equipment which are connected to smart controllers.

(2) The electricity utilization controller has advantages of multiple protection functions, high precision computation and reliability, strong practicability and intelligence, low cost, etc., which can effectively reduce users' electricity costs and help users save energy effectively, thus has certain application prospect in the market.

(3) The next research direction of this paper is focus on developing a complete set of intelligent home electricity utilization simulative system based on the designed smart controller in this paper, the system includes full rigged home electrical equipment, smart interactive terminals (smart phones, tablets, etc.), intelligent APP application software, smart hone LAN transmission and communication network based on ZigBee, as well as distributed photovoltaic power source with small capacity and the corresponding inverter controllers, meanwhile, the home energy efficiency management mechanism will be established, and the changing situations of all kinds of electric load will be synthetically considered. The real-time electricity price and home energy efficiency analysis results will be given by the system and the energy efficiency energy-saving scheme is presented as well, meanwhile, all kinds of electricity utilization equipment and distributed energy access will be managed in uniformity, thus the smart home electricity utilization direction can be transferred from passive acceptance to active management, finally realize interaction with the grid, and lay a good foundation for the final establishment of a strong active power distribution system.

\section{References}

[1] Mingtian Fan, Zuping Zhang, Aoxue Su, et al. , Enabling technologies for active distribution systems, Proceedings of the CSEE (In Chinese), 33 (22): 12-18+5, 2013.

[2] Bo Zhao, Caisheng Wang, Jinhui Zhou, et al. , Technology and its trends of active distribution network, Automation of Electric Power Systems (In Chinese), 38(18): 125-135, 2014.

[3] Yi You, Dong Liu, Wenpeng Yu, et al. , Technology and its trends of active distribution network, Automation of Electric Power Systems (In Chinese), 36(18): 10-16, 2012.

[4] Xinxin Gu, Xuesong Lin, Chunjia Liu, et al. , Technology and practice of intelligent equipment of active distribution network, Distribution \& Utilization (In Chinese), 31(1): 42-44, 2014.

[5] Honglin Ma, New remote control socket design and research, Mechanical \& Electrical Engineering Magazine (In Chinese), 20(5): 51-52, 2003.

[6] Gan Zhang, The study and application of communication system for distribution automation, Chinese Master's Degree Paper, Zhengzhou University, Zhengzhou, China, 2011.

[7] Xinxing Zhang, The design of smart outlet based on Android phone, Chinese Master's Degree Paper, Zhejiang University, Hangzhou, China, 2014.

[8] Juan Wang, Design and implementation of the smart home system based on ZigBee wireless sensor networks, Chinese Master's Degree Paper, East China Institute of Technology, Nanchang, China, 2013.

[9] Maosheng Qin, The design of smart home based on ZigBee, Chinese Master's Degree Paper, Taiyuan University of Technology, Taiyuan, China, 2011.

[10] Yu Dong, Design and implementation of domestic smart electricity consumption device, Chinese Master's Degree Paper, Zhejiang University, Hangzhou, China, 2013.

[11]Hua Liu, Research and design of the system of collecting farming information, Chinese Master's Degree Paper, Taiyuan University of Technology, Taiyuan, China, 2007. 